\title{
Monitoring of Anthrax Prevalence in Georgia 2009-2017
}

\section{Lela Kerdzevadze}

Administrative, Laboratory of the Ministry of Agriculture, Tbilisi, Georgia

Objective

One of the main objectives of these studies was to improve Anthrax laboratory diagnostics in order to properly monitor the prevalence and distribution of the disease in Georgia. For this geographic information system (GIS) was implemented and used as the additional tool to the laboratory tests for better visualization, summary results and risk assessment.

\section{Introduction}

Anthrax is an acute infectious disease of historical importance caused by Bacillus anthracis (B. anthracis), a spore-forming, soilborne bacterium with a remarkable ability to persist in the environment. Anthrax is endemic in many countries, including Georgia. Laboratory of the Ministry of Agriculture (LMA) has been actively working on the disease science 1907 and constantly improving diagnostics. In 2009-2017 the laboratory participated in cooperative biological studies. One of the main objectives of these studies was to improve Anthrax laboratory diagnostics in order to properly monitor the prevalence and distribution of the disease in Georgia.

\section{Methods}

In 2009 -2011, within GG18, LMA tested 130(animal and environmental) anthrax suspected samples collected from different regions of Georgia. Later, in 2014 - 2017, studies (TAP7; GG27) were focused on soil sample collection and 2825 specimens were collected from the entire country.

Samples were tested according to Treat Agent Detection and Response (TADR) algorithm following standard operation procedures (SOPs). Cultures were isolated through Bacteriology tests - Gram strain, Lysis by gamma phage, Motility test, DFA and confirmed by Molecular Biology (PCR).

In 2009, within the studies, geographic information system (GIS) was implemented and used as the additional tool to the laboratory tests for better visualization, summary results and risk assessment.

\section{Results}

Totally, 2955 collected samples were tested. 86 cultures were isolated and confirmed. The results - anthrax cases were mapped by regions, rayons and villages, also positive cases were mapped by sample type and course, Majority of positive cases were in Kvemo Kartli (53\%), 19\% were from Kakheti, 19\% - from Imereti and less distributed in other regions.

Applying modern GIS the final map of anthrax foci in Georgia was created including both - old (historical data) and new (recent data) foci.

\section{Conclusions}

The studies aimed to improve Anthrax laboratory diagnostic in Georgia. Better Laboratory diagnostic with modern GIS analysis supports the monitoring of the disease prevalence in Georgia and significantly improves public health system in the country. 